\title{
A Psicologia Evolucionista e os Domínios da Cognição Social
}

\author{
Silvio José Lemos Vasconcellos ${ }^{1}$ \\ Faculdades Integradas de Taquara \\ Pontificia Universidade Católica do Rio Grande do Sul \\ Antonio Jaeger \\ Pontifícia Universidade Católica do Rio Grande do Sul \\ Maria Alice Parente \\ Cláudio Simon Hutz \\ Universidade Federal do Rio Grande do Sul
}

\begin{abstract}
RESUMO - O estudo da cognição social começou a ter destaque com o surgimento da Psicologia Cognitiva no final dos anos 60 e início dos anos 70. A expressão refere-se aos processos que orientam o comportamento diante de outros indivíduos. Este trabalho analisa uma nova abordagem da cognição social baseada na Psicologia Evolucionista. Os autores descrevem alguns aspectos relacionados à filogênese da cognição humana. A revisão bibliográfica desta pesquisa considerou livros e artigos sobre o tema publicados no período de 1975 a 2006. O artigo explica também os principais mecanismos que se apresentam como produtos da seleção natural e que surgiram para resolver problemas da vida social na história evolutiva humana. Na parte final, discute-se a importância desse novo enfoque.
\end{abstract}

Palavras-chave: psicologia evolucionista; cognição social; evolução.

\section{Evolutionary Psychology and the Social Cognition Domains}

\begin{abstract}
The study of social cognition came to prominence with the rise of Cognitive Psychology in the late 1960s and early 1970s. The expression refers to the processes that subserve behavior in response to other individuals. This paper analyses a new approach of social cognition based on Evolutionary Psychology. The authors describe some aspects related to the phylogeny of human cognition. The bibliographical review of this research considered the 1975-2006 period and included articles and books about this subject. The present paper also explains the main mechanisms that are products of natural selection and that appeared to solve social life problems in human evolutionary history. In the last part, the importance of this approach is discussed.
\end{abstract}

Keywords: evolutionary psychology; social cognition; evolution.

O ser humano, na sua forma atual, apresenta-se como o produto de um longo processo evolutivo. Conforme salienta Zimmer (2003), o tempo decorrente desde que os nossos ancestrais abandonaram as práticas de coleta e caça representa menos de $1 \%$ de toda a história dos hominídeos. Esse é, indubitavelmente, um tempo muito curto para que nele possamos identificar a origem de todas as inúmeras capacidades mentais humanas. Nesse sentido, a ideia de que a mente é também o resultado de um processo evolutivo que se encarregou de favorecer uma série de capacidades cognitivas capazes de conferir novas vantagens adaptativas é, na atualidade, quase um consenso.

De um modo mais específico, no entanto, a Psicologia Evolucionista não se apresenta apenas como uma nova abordagem disposta a defender a evolução do intelecto humano. Pode-se afirmar que os psicólogos evolucionistas acabam indo além dessa concepção, ao preconizarem que a mente é um conjunto de funções cuja emergência resulta da própria necessidade de encontrar soluções específicas para problemas específicos. Mais do que atender a propósitos gerais, a cognição do Homo sapiens foi moldada, de acordo com essa

1 Endereço para correspondência: Av. Oscar Martins Rangel, 4500. Taquara, RS. CEP 95600-000.E-mail: silviojlvasco@hotmail.com. perspectiva, para gerar e manter padrões adaptativos e diversificados de interação com o meio e com os outros indivíduos. Nesses termos, uma das analogias mais recorrentes para os psicólogos evolucionistas é a de que a mente funciona como um "canivete suíço", ou seja, abarca um conjunto de funções especializadas que, por sua vez, coexistem em um mesmo aparato (Cartwright, 2001; Evans \& Zarate, 1999).

Uma vez que a socialização fundamentou, de um modo mais abrangente, o próprio desenvolvimento do intelecto humano, o estudo da denominada cognição social passou a ser um dos pilares da Psicologia Evolucionista. Mais do que considerar as pressões evolutivas que favoreceram a sofisticação de determinadas funções cognitivas, tais como raciocínio, memória e linguagem, essa abordagem procura elucidar uma série de mecanismos sutis e direcionados para alguns aspectos mais específicos da interação social.

Em termos gerais, este artigo procura sintetizar e discutir os principais preceitos relativos ao desenvolvimento da cognição social com base nos pressupostos da Psicologia Evolucionista. A revisão apresentada contribui para a elucidação das principais asserções no que se refere a uma compreensão evolucionista de uma das facetas mais relevantes da cognição humana. Inicialmente, os autores explicam o próprio conceito de cognição social para então explanar a forma como essa é 
compreendida a partir da citada abordagem. Na parte final deste estudo, os autores discorrem sobre as perspectivas dessa nova área de estudo, considerando o estado atual de conhecimento sobre o assunto.

\section{O Conceito de Cognição Social}

Uma vez que o desenvolvimento cognitivo, em suas diferentes modalidades, está relacionado à capacidade de interação social do indivíduo, pode-se denominar de cognição social o conjunto de processos cognitivos que tornam possível o desenvolvimento social humano. Dentro dessa esfera específica da cognição, não estariam incluídos os processos empregados na consolidação do pensamento estritamente lógico de nível simbólico. No que se refere a essa questão, Flavell, Miller e Miller (1999) salientam que:

A cognição social toma os humanos e seus afazeres como sujeitos; ela significa a cognição sobre pessoas e suas ações. As máquinas, a matemática e os julgamentos morais são objetos e produtos da cognição humana, por exemplo, mas somente os últimos seriam considerados um tópico dentro da cognição social humana. A cognição social lida com o mundo estritamente social, não com os mundos físicos e lógico-matemático, embora todos os três tenham as marcas do engenho humano. (p. 145)

Os primeiros estudos sobre cognição social datam do final dos anos 60 e início dos anos 70. De um modo mais amplo, Lamb e Sherrod (1981) postularam que a cognição social refere-se à forma pela qual o indivíduo percebe e compreende outras pessoas. Fu, Goodwin, Sporakowki e Hinkle (1987) acrescentam a noção de que o próprio conceito abarca a capacidade de integrar perspectivas. Nessa mesma linha, Bugental (2000) salienta que tal capacidade torna-se viável por intermédio da execução de um algoritmo da vida social. Fiske e Taylor (1991) inserem nessa perspectiva a cognição sobre si, considerando, além do modo como os indivíduos pensam sobre os outros, também o modo como concebem o próprio pensamento diante dos outros. Schneider (1991) assevera ainda a importância de diferenciar estímulos que podem ser considerados sociais de outros tipos de estímulos que não se inserem nessa categoria.

Ao destacar os estudos da cognição social como um conjunto de abordagens voltado não apenas para o modo de investigar, mas também de conceber o desenvolvimento psíquico, Ramires (2003) destaca que a cognição social reconhece a criança ativa e interativa, postulando um papel construtivo nesse processo. Ainda sobre as questões de desenvolvimento, a autora menciona que as pesquisas relevantes nesse campo abordam o próprio desenvolvimento da capacidade de identificar, recordar e reconhecer objetos sociais, fazendo distinção com os objetos inanimados do ambiente (Ramires, 2003)

O modelo de processamento de informações sociais de Crick e Dodge (1994) exemplifica uma das abordagens que integram esse amplo campo de investigação e considera uma série de processos de atribuição, julgamento social e autopercepção. De acordo com o modelo exposto, certos déficits nos diferentes mecanismos responsáveis pelo processamento das informações sociais acabam por sustentar um padrão de comportamentos agressivos, na medida em que se verifica um prejuízo na própria habilidade de resolver situações de impasse que tendem a ocorrer no dia-a-dia. Nesse sentido, uma série de trabalhos empíricos demonstrou que crianças e adolescentes avaliados como sendo mais agressivos que seus pares em grupos controles acabam por apresentar tendências específicas em termos de processamento das informações sociais (Dodge, 1993; Dodge \& Frame, 1982; Dodge \& Newman, 1981; Dodge \& Somberg, 1987) De um modo geral, pode-se dizer que esse modelo propõe um entendimento sobre o modo como o sujeito irá se servir dos dados sociais a fim de gerar comportamentos específicos (Dodge, Laird, Lochman \& Zelli, 2002).

Em uma perspectiva neurobiológica, Damasio (2003) afirma que várias estruturas cerebrais têm um papel chave no controle de condutas e capacidades sociais. Dentre elas, pode-se destacar o córtex pré-frontal ventromedial, o córtex somatosensorial direito, a amígdala e a ínsula. $\mathrm{O}$ córtex pré-frontal ventromedial estaria envolvido com o raciocínio social e com a tomada de decisões; a amígdala, com o reconhecimento emocional das expressoes faciais; o córtex somatosensorial direito, com a capacidade de simular e sentir empatia e a ínsula, com a resposta autonômica (Bechara, Damasio \& Damasio, 2003).

No que se refere aos seus aspectos estruturais e funcionais, pode-se dizer que a cognição social abarca a ampla capacidade de gerar interpretações e respostas diante dos signos sociais. A emergência e sofisticação dessa capacidade nos seres humanos não encontram, por certo, equiparações quando se considera o desenvolvimento das demais espécies. Na sequência deste artigo, serão analisados os principais aspectos relacionados à filogênese da própria cognição social, a partir de pressupostos mais gerais a respeito da evolução dos hominídeos.

\section{Evolução, Cérebro e Cognição}

A evolução das capacidades mentais humanas não é, por certo, apenas uma questão de tamanho cerebral. Ainda que a própria expansão cerebral tenha sido favorecida pela seleção natural, o tamanho relativo mostra-se mais importante (Gould, 1988). A noção de coeficiente de encefalização ocupa, nesse sentido, um papel central para que possamos compreender a evolução do intelecto. Considera-se, a partir dela, o tamanho do cérebro em termos relativos ao próprio tamanho corporal da espécie (Cunha, 2003).

Segundo Mithen (2002), a evolução dos primatas data de 56 milhões de anos. Há aproximadamente sete milhões de anos, hominídeos e chimpanzés compartilhavam um ancestral comum (Diamond, 1992). No que se refere à análise cladística das duas espécies, não resta dúvida, no entanto, quanto ao fato de existir significativas diferenças entre ambas, incluindo o próprio coeficiente de encefalização. A capacidade cerebral dos seres humanos modernos atingiu um patamar único, sendo que diferentes fatores podem ter contribuído para isso.

Uma vez que cérebros maiores consomem mais energia, uma mudança de hábitos alimentares pode explicar um sig- 
nificativo aumento do tamanho cerebral ocorrido na linha homo, separando diferentes espécies Australopitecus dos primeiros Homo habilis. Além disso, conforme salienta Eccles (1989), a postura bípede pode ter influenciado a destreza manual que, por sua vez, também influenciou o aparecimento da linguagem em seus aspectos mais primordiais.

Além dos fatores destacados, considera-se ainda a elevada importância do convívio social para a evolução cognitiva do Homo sapiens. Dunbar (1992) denominou "grupos cognitivos" o conjunto de indivíduos sobre os quais podemos ter informações sociais. $\mathrm{O}$ autor examinou fósseis cranianos de diferentes espécies de hominídeos, observando um aumento quanto ao número de indivíduos integrantes desses mesmos grupos. Os primeiros bandos de Australopitecus seriam formados por aproximadamente 67 indivíduos. Na espécie Homo habilis tais bandos incluiriam uma média de 82 indivíduos, chegando a atingir 100 indivíduos na espécie Homo erectus (Dunbar, 1996). De acordo com essa perspectiva, grupos maiores representam uma necessidade maior de armazenar e processar informação. Uma correlação positiva entre a capacidade do neocórtex e o tamanho dos grupos nos quais os indivíduos tendem a estar inseridos também é verificada em diferentes espécies de primatas (Dunbar, 1992).

O convívio social demonstra ser, portanto, um dos fatores fundamentais para a sofisticação da inteligência. Embora, conforme ressalte Tobias (1997), não tenha ocorrido uma única frente de hominização, essa lógica perpassa a própria evolução dos hominídeos que culminou no aparecimento dos primeiros humanos modernos há aproximadamente $170 \mathrm{mil}$ anos (Zimmer, 2003). A interação social é, nesses termos, uma das questões centrais para compreender a evolução da mente humana e, segundo a Psicologia Evolucionista, um dos pontos de partida para descrevê-la a partir das suas especializações.

\section{Cognição Social e Psicologia Evolucionista}

De um modo geral, a Psicologia Evolucionista tem procurado rever alguns achados da Sociobiologia, tecendo, em certos casos, críticas a algumas de suas generalizações (Pinker, 2004). Essa abordagem precedente buscou investigar as raízes da cultura, considerando o seu suporte biológico. Autores como Hamilton e Wilson postularam que o comportamento social humano, nas suas mais diferentes esferas, pode ser explicado a partir de necessidades adaptativas vinculadas a um processo de seleção natural capaz de atingir também inúmeras funções mentais (Hamilton, 1964).

De um modo mais específico, a Psicologia Evolucionista procura descrever uma série de mecanismos mentais vinculados a esse mesmo processo adaptativo, buscando ainda respostas conciliatórias diante do aparente problema natureza versus criação (Pinker, 2004). De acordo com esse entendimento, não faz sentido opor as aquisições culturais a uma possível base genética para o comportamento humano, uma vez que a cultura também representa uma via para a consolidação de determinadas tendências biológicas (Cosmides \& Tooby, 1989). Conforme ressalta Ridley (2004), a abordagem evolucionista fica "tão à vontade com explicações de criação, como com as explicações da natureza" (p. 309).
Nesses termos, não há uma negação quanto à própria realidade biopsicossocial que perfaz o ser humano.

A partir desse entendimento, Cosmides (1989), ao utilizar diferentes versões do teste de Wason, demonstrou, por exemplo, que a capacidade lógica humana pode ser melhor utilizada em situações sociais do que em situações não sociais. Esse postulado demonstra ser um ponto de partida para compreendermos que, sob diferentes aspectos, podem existir especificidades na forma como nossos recursos cognitivos são alocados nos processos de interação. Para Tooby e Cosmides (1990), o desenvolvimento de estruturas cognitivas responsáveis por um processamento mais especializado de informações sociais surge em diferentes etapas do desenvolvimento ontogenético, ainda que esteja vinculado a uma base filogenética.

Nesses termos, o entendimento da cognição social abarca, segundo a citada abordagem, explicações sobre o modo como o comportamento humano decorre da própria necessidade de propagar genes, sem que isso represente um impedimento em termos de convívio entre pares. Em outras palavras, ainda que o fundamento básico da teoria da evolução seja a competição entre indivíduos, comportamentos cooperativos fundamentados numa lógica de custo e benefício também acabaram sendo favorecidos pela seleção natural (Oliva \& cols., 2006). Desse modo, mecanismos distintos atrelados à cognição social emergiram como estratégias evolutivamente estáveis, mantendo possibilidades de propagação e coexistência.

A mente social dos humanos modernos comporta, segundo a abordagem evolucionista, uma série diversificada de tendências inatas, uma vez que a vida em sociedade viabiliza diferentes formas de interação. $\mathrm{O}$ indivíduo que está, por exemplo, envolvido em uma situação de negociação não age do mesmo modo que alguém que possa estar cortejando outro indivíduo do sexo oposto. Uma pessoa que presta socorro para outra protagoniza a ação de mecanismos distintos daqueles que seriam empregados caso estivesse tentando subjugar alguém. Desse modo, a analogia do "canivete suíço" parece fazer sentido. Afinal, a partir dela, considera-se a possibilidade de que um conjunto de mecanismos mentais possa, ao invés de um único mecanismo mental, conferir maiores vantagens adaptativas para os organismos que os executam. A vida em sociedade mostrou-se capaz de fundamentar um processo de evolução cognitiva, bem como a complexidade das relações decorrentes desse mesmo processo. Na próxima parte do artigo, serão analisados os principais mecanismos e os respectivos domínios que integram a cognição social do Homo sapiens.

\section{Os Principais Domínios da Cognição Social}

Uma vez que a vida social comporta diferentes tipos de situações de interação, uma série de mecanismos distintos são constituintes da cognição social humana. A Psicologia Evolucionista vem procurando, cada vez mais, identificar e descrever esses mesmos mecanismos. Para tanto, faz-se necessário considerar também os conceitos de causas finais e causas próximas. As causas finais dizem respeito a explicações associadas à evolução e as causas próximas aludem, 
por sua vez, processos biológicos que operam no organismo no momento em que o comportamento pode ser observado (Pervin \& John, 2004).

Postular vantagens adaptativas para uma determinada tendência do comportamento não equivale a afirmar que essa mesma tendência seja igualmente favorecida pela seleção natural nas condições atuais de sobrevivência do organismo. Mostra-se importante considerar a própria história evolutiva da espécie. A mente humana foi sendo moldada, de acordo com esse entendimento, por pressões seletivas totalmente alheias ao próprio avanço tecnológico e cultural que se verifica na atualidade. Sendo assim, compreender as diferentes peculiaridades da cognição social com base numa perspectiva evolucionista representa também uma necessidade de verificar a pertinência de determinados mecanismos mentais diante do contexto no qual esses mecanismos emergiram.

Em termos mais gerais, Schaller, Park e Kenrick (2006) destacam que os principais mecanismos cuja função é sustentar determinadas tendências do comportamento direcionadas à vida em sociedade inserem-se em seis domínios distintos denominados, por sua vez, de domínios de interação. $\mathrm{O}$ primeiro deles refere-se à formação de alianças e vincula-se à capacidade humana de formar grupos cooperativos com outros indivíduos. A busca pela obtenção e manutenção de status dentro do grupo constitui-se no segundo domínio descrito pelos autores. $\mathrm{O}$ terceiro domínio diz respeito à constante necessidade de auto-proteção, considerando a ameaça de terceiros, bem como a busca por recursos que garantam a sobrevivência pessoal. O quarto nível descreve a procura por parceiros sexuais, sendo que o nível seguinte refere-se ao conjunto de mecanismos voltados para manter a relação com esses mesmos parceiros. No sexto e último nível, insere-se o cuidado com a prole, representado pela busca de bem estar e proteção para os próprios descendentes.

Capacidades humanas que atuam de um modo mais generalizado não constituem, segundo Schaller, Park e Kenrick (2006), um nível específico, mas, de outro modo, viabilizam a consolidação dos diferentes aspectos da cognição social. É o caso, por exemplo, da capacidade humana de teorizar sobre os estados mentais alheios, denominada teoria da mente (Gerrans, 2002). Essa capacidade fundamenta uma série de mecanismos adaptativos na espécie que dependem da possibilidade de conjeturar sobre as expectativas e sobre os interesses alheios (Gerrans, 2002). Nesse sentido, formar alianças, buscar status dentro de um grupo, autoproteger-se, procurar e manter parceiros sexuais e, até mesmo, zelar pela sobrevivência dos filhos representam instâncias de interação nas quais a capacidade de compreender a mente do outro se revela necessária.

De um modo geral, os domínios da cognição social propostos por Schaller, Park e Kenrick (2006) abarcam inúmeros mecanismos que já vêm sendo estudados pelos psicólogos evolucionistas. Uma vez que a evolução do intelecto humano mostra-se tributária das relações grupais que foram sendo consolidadas ao longo da evolução dos hominídeos, a cognição social comporta mecanismos distintos. Conforme salientado, a mente humana demonstra ser um conjunto de soluções específicas para problemas específicos. A coexistência de mecanismos sutis direcionados para uma série de propósitos específicos revela-se, portanto, um pressuposto norteador para os estudos da cognição social desenvolvidos no âmbito da Psicologia Evolucionista.

\section{Considerações Finais}

Embora os primeiros estudos voltados diretamente para a cognição social humana tenham ocorrido há mais de três décadas, a Psicologia Evolucionista, mais recentemente, fundamentou uma nova maneira de compreender esse fenômeno (Cosmides \& Tooby, 1992). Para a citada abordagem, mais do que delimitar algumas funções cognitivas envolvidas no campo da cognição social, mostra-se necessário explicar a razão de ser das mesmas, considerando a nossa própria história evolutiva. Dito de outro modo, faz-se necessário entender que uma série de mecanismos mentais recorrentes nas situações de interação social não são meros produtos do acaso.

Conforme os pressupostos destacados neste artigo, mecanismos desse tipo inserem-se em diferentes níveis de interação e atendem propósitos distintos. Nesse sentido, a tendência para a realização de comportamentos altruístas pode, portanto, coexistir com outras tantas tendências menos "nobres". Diante disso, mostra-se importante evitar que as análises incorram em uma falácia naturalista (Pinker, 2004). Ou seja, afirmar que um mecanismo mental foi favorecido por pressões seletivas que se consolidaram ao longo de muito tempo, não equivale a afirmar que esses mesmos mecanismos devam servir de parâmetros para as ações na sociedade atual. A seleção natural é simplesmente um processo que depurou tendências comportamentais e não uma entidade encarregada de programar rotinas fixas para as condutas humanas. Nesse sentido, todo e qualquer avanço referente a essa área de estudo deve estar pautado numa discussão sobre os pressupostos éticos quanto aos próprios achados científicos e suas respectivas implicações para a sociedade. Essa é uma problemática que deve continuar sendo verdadeiramente abordada no âmbito da Psicologia Evolucionista.

Com base nessas considerações, pode-se enfatizar a pertinência da abordagem evolucionista para as ciências que estudam o comportamento humano e, sobretudo, para aquelas que estudam os comportamentos na esfera social. Compreender a evolução da mente, a partir da necessidade de sofisticar ou abolir certos padrões comportamentais ao longo da história evolutiva dos hominídeos, demonstra ser um ponto de partida para explorar outras peculiaridades da vida em sociedade. O enfoque evolucionista pode, portanto, subsidiar novos estudos sobre a cognição social humana, vinculados, por sua vez, aos diferentes campos do conhecimento humano. Para tanto, mostram-se necessários novos trabalhos que possam investigar os diferentes níveis desse construto, ou mesmo, melhor explicar a inter-relação dos mecanismos que o constituem.

\section{Referências}

Bechara, A., Damasio, H., \& Damasio, A. (2003). Role of the amygdala in decision-making. Annals of the New York Academy of Sciences, 985, 359-369. 
Bugental, D. B. (2000). Acquisition of the algorithms of social life: A domain-based approach. Psychological Bulletin, 126, 187-219.

Cartwright, J. H. (2001). Evolutionary explanations of human behaviour. New York: Routledge.

Cosmides, L. (1989). The logic of social exchange: Has natural selection shaped how humans reason? Studies with the Wason selection task. Cognition, 31, 187-276.

Cosmides, L., \& Tooby, J. (1989). Evolutionary psychology and the generation of culture, Part II. Case study: A computational theory of social exchange. Ethology and Sociobiology, 10, 51-97.

Cosmides, L., \& Tooby, J. (1992). Cognitive adaptation for social exchange. Em J. Brkow, L. Cosmides, \& Tooby, J. (Orgs.), The adapted mind (pp. 163-228). New York: Oxford University Press.

Crick, N. R., \& Dodge, K. A. (1994). A review and reformulation of social information-processing mechanisms in children's social adjustment. Psychological Bulletin, 115, 74-101.

Cunha, E. (2003). As capacidades cognitivas na evolução humana. Em G. J. Gauer \& Machado, D. S. (Orgs), Filhos e vítimas do tempo da violência - a família, a criança e o adolescente (pp. 13-31). Curitiba: Juruá.

Damasio, A. (2003). Feelings of emotion and the self. Annals of the New York Academy of Sciences, 1001, 253-261.

Diamond, J. M. (1992). Evolutionary physiology: The red flag of optimality. Nature, 355, 204-206.

Dodge, K. A. (1993). Social-cognitive mechanisms in the development of conduct disorder and depression. Annual Review of Psychology, 44, 559-584.

Dodge, K. A., \& Frame, C. L. (1982). Social cognitive biases and deficits in aggressive boys. Child Development, 53, 620-635.

Dodge, K. A., Laird, R., Lochman, J. E., \& Zelli, A. (2002). Multidimensional latent-construct analysis of children's social information processing patterns: Correlations with aggressive behavior problems. Psychological Assessment, 14, 60-73.

Dodge, K. A., \& Newman, J. P. (1981). Biased decision making processes in aggressive boys. Journal of Abnormal Psychology, 90, 375-379.

Dodge, K. A., \& Somberg B. R. (1987). Hostile attributional biases among aggressive boys are exacerbated under conditions of threats to the self. Child Development, 58, 213-224.

Dunbar, R. I. M. (1996). Grooming, gossip and the evolution of the language. Londres: Faber \& Faber.

Dunbar, R. I. M. (1992). Neocortex size as a constraint on group size in primates. Journal of Human Evolution, 20, 469-493.

Eccles, J. (1989). A evolução do cérebro: a criação do eu. Lisboa: Instituto Piaget.

Evans, D., \& Zarate, O. (1999). Introducing evolutionary psychology. Cambridge: Icon Books.

Fiske, S. T., \& Taylor, S. E. (1991). Social cognition (2 ${ }^{\mathrm{a}}$ ed.). New York: McGraw-Hill.

Flavell, J. H., Miller, P., \& Miller, S. A. (1999). Desenvolvimento cognitivo. Porto Alegre: Artmed.
Fu, V. R., Goodwin, M. P., Sporakowki, M. J., \& Hinkle, D. E. (1987). Children's thinking about familiy characteristes and parent attributes. Journal of Genetic Psychology, 148, 153-166.

Gerrans, P. (2002). The theory of mind module in evolutionary psychology. Biology and Philosophy, 17, 305-321.

Gould, S. J. (1988). O mundo depois de Darwin. Lisboa: Presença.

Hamilton, W. D. (1964). The genetical theory of social behavior: I and II. Journal of Theoretical Biology, 7, 1-52.

Lamb, M. E., \& Sherrod, L. R. (1981). Infant social cognition: Empirical and theoretical considerations. New Jersey: Lawerence Erlbaum.

Mithen, S. (2002). A pré-história da mente: uma busca das origens da arte, da religião e da ciência. São Paulo: UNESP.

Oliva, A. D., Otta, E., Ribeiro, F. L., Bussab, V. S. R., Lopes, F. A., Yamamoto, M. E., \& Moura, M. L. S. (2006). Razão, emoção e ação em cena: a mente humana sob um olhar evolucionista. Psicologia: Teoria e Pesquisa, 22, 53-61.

Pervin, L. A., \& John, O. P. (2004). Personalidade: teoria e pesquisa. Porto Alegre: Artmed.

Pinker, S. (2004). Tabula rasa: a negação contemporânea da natureza humana. São Paulo: Companhia das Letras.

Ramires, V. R. R. (2003). Cognição social e teoria do apego: possíveis articulações. Psicologia, Reflexão e Crítica, 16, 403-410.

Ridley, M. (2004). O que nos faz humanos: genes, natureza e experiencia. Rio de Janeiro: Record.

Schaller, M., Park, J. H., \& Kenrick, D. T. (2006). Human evolution and social cognition. Em R. I. M. Dunbar \& L. Barrett (Eds.), Handbook of evolutionary psychology. Oxford: Oxford University Press.

Schaller, M, Park, J. H., \& Faulkner, J. (2003). Prehistoric dangers and contemporary prejudices. European Review of Social Psychology, 14, 105-37.

Schneider, D. J. (1991). Social cognition. Annual Review of Psychology, 42, 527-561.

Tobias, P. V. (1997). Evolution of brain size, morphological restructuring and longevity in early hominids Em S. U. Dani, A. Hori \& G. F. Walter (Eds.), Principals of neural aging. Amsterdam: Elseiver.

Tooby, J., \& Cosmides, L. (1990) On the universality of human nature and the uniqueness of the individual: The role of genetics and adaptation. Journal of Personality, 58, 17-67.

Zimmer, C. (2003). O Livro de ouro da evolução. Rio de Janeiro: Ediouro. 